\title{
Exercise-mediated locomotor recovery and lower-limb neuroplasticity after stroke
}

\author{
Larry W. Forrester, PhD; ${ }^{1-2 *}$ Lewis A. Wheaton, PhD; ${ }^{3}$ Andreas R. Luft, MD $^{4}$ \\ ${ }^{1}$ Department of Veterans Affairs (VA) Maryland Health Care System, Research Service, Baltimore, MD; ${ }^{2}$ Department \\ of Physical Therapy and Rehabilitation Science, University of Maryland School of Medicine, Baltimore, MD; ${ }^{3} V A$ \\ Maryland Health Care System, Baltimore, MD; ${ }^{4}$ Hertie Brain Institute for Clinical Brain Research, University of \\ Tübingen, Tübingen, Germany
}

\begin{abstract}
Assumptions that motor recovery plateaus within months after stroke are being challenged by advances in novel motor-learning-based rehabilitation therapies. The use of lowerlimb treadmill (TM) exercise has been effective in improving hemiparetic gait function. In this review, we provide a rationale for treadmill exercise as stimulus for locomotor relearning after stroke. Recent studies using neuroimaging and neurophysiological measures demonstrate central nervous system (CNS) influences on lower-limb motor control and gait. As with studies of upper limbs, evidence shows that rapid transient CNS plasticity can be elicited in the lower limb. Such effects observed after short-term paretic leg exercises suggest potential mechanisms for motor learning with TM exercise. Initial intervention studies provide evidence that long-term TM exercise can mediate CNS plasticity, which is associated with improved gait function. Critical needs are to determine the optimal timing and intensities of TM therapy to maximize plasticity and learning effects.
\end{abstract}

Key words: gait, gait training, hemiparesis, locomotor, lower limb, motor control, motor learning, neuroplasticity, neurorehabilitation, rehabilitation, stroke, treadmill exercise.

\section{INTRODUCTION}

Approximately 700,000 strokes occur annually in the United States; 50 percent of the 550,000 survivors experience residual hemiparesis and approximately 165,000 of those individuals have mobility deficits requiring assistance with walking [1-3]. In this population, hemiparetic gait is a major problem that limits mobility, increases risk of falls, and imposes higher energy demands for basic daily activities [4-5]. Gait deviations due to hemiparesis are well documented, in terms of both clinical manifestation and biomechanical analyses [6-7]. Classic models of stroke recovery indicate that improvements in both upper- and lower-limb motor function plateau between 3 and 6 months poststroke [8]. Recent studies have challenged this assumption by suggesting that specific training interventions that target use of the hemiparetic limbs can improve motor control and neural plasticity. The research community now

\footnotetext{
Abbreviations: CNS $=$ central nervous system, $\mathrm{EMG}=$ electromyography, FES = functional electrical stimulation, fMRI = functional magnetic resonance imaging, $\mathrm{M} 1=$ primary motor cortex, MEP = motor-evoked potential, $\mathrm{MRCP}=$ movementrelated cortical potentials, NIRS $=$ near-infrared spectroscopy, PAS = paired associative stimulation, PBWS = partial bodyweight suspension, RR\&D = Rehabilitation Research and Development, $\mathrm{S} 1$ = primary somatosensory cortex, $\mathrm{SCI}=$ spinal cord injury, SMA = supplementary motor area, SMC = sensory motor cortex, $\mathrm{TA}=$ tibialis anterior, $\mathrm{T}-\mathrm{AEX}=$ treadmill aerobic exercise, $\mathrm{TM}=$ treadmill, $\mathrm{TMS}=$ transcranial magnetic stimulation, VA = Department of Veterans Affairs.

*Address all correspondence to Larry W. Forrester, PhD; University of Maryland School of Medicine, 100 Penn Street, Suite 115, Baltimore, MD 21201-1082; 410-706-5212; fax: 410-706-6387. Email: Iforrester@som.umaryland.edu DOI: 10.1682/JRRD.2007.02.0034
} 
widely accepts that the central nervous system (CNS) comprises inherently plastic neural networks that are continuously amenable to reorganization in the service of functional behaviors [9]. As a consequence, new therapeutic approaches seek to exploit experience-based CNS plasticity to mediate functional improvements. A common thread among most of these interventions is an adherence to the principles of motor learning, as defined by incorporating high volumes of task-oriented practice along with the added dimensions of goal setting and performance feedback [10].

Studies of therapies that improve function and induce neuroplasticity in hemiparetic upper limbs in human survivors of stroke have supported an emerging focus on developing new learning-based strategies for improving gait and balance function in individuals with lower-limb hemiparesis after stroke [11-17]. Here we review evidence that one particular mode of exercise, treadmill (TM) training as applied in a number of approaches, can be employed to improve gait function in survivors of stroke with residual hemiparesis. We will suggest that basic motor learning strategies can alter underlying neural mechanisms to improve hemiparetic function of the lower limb and may also be effective in recovery of walking ability after stroke. Following a brief overview of the rationale and early results from studies using TM training with stroke, we provide examples that illustrate the role of the CNS in lower-limb motor control and gait. Our focus then shifts to an overview of how the neurophysiology of lower-limb motor control is sensitive to short-term adaptations and rapid plasticity. Finally, we review the early evidence of central neuroplasticity underlying lower-limb function and gait using long-term TM training protocols.

\section{RATIONALE FOR TREADMILL LOCOMOTOR LEARNING AFTER STROKE}

Findings from spinalized animal models demonstrate that walking without supraspinal inputs can occur when the animal is placed on a moving TM [18]. Thus, several investigations have studied TM training as a means to improve locomotor function in subjects with incomplete spinal cord injury (SCI) and stroke. Visintin et al. first adapted the findings from spinalized animals to human experiments, reasoning that activation of subcortical neural structures by TM walking could provide a physiologi- cal stimulus for recovery of gait function [19-20]. These studies support the rationale that TM-generated stepping patterns in neurologically injured humans can help deliver repetitive sensory inputs to the spinal cord, which in turn could mediate locomotor learning and neural plasticity through a process of sensory motor integration [21]. Additional feasibility for this idea was shown in a study of the immediate effects of the TM stimulus on hemiparetic gait patterns in naïve subjects with chronic stroke [22]. While controlling for walking speed, paretic limb stance-swing parameters and loading impulse immediately became more symmetrical on the TM compared with usual overground walking. Analyses of electromyography (EMG) activation patterns showed that this symmetry was not an artifact of TM-induced mechanical perturbations, as timing of EMG bursts shifted significantly within the paretic gait cycle [23]. Thus, untrained individuals with hemiparesis can alter how they walk during a brief exposure to the TM stimulus. A question then arises as to whether an adequate amount of practice would promote lasting changes in their gait function. If so, is the effect reflected in measures of lower-limb motor control and central neural plasticity?

\section{TREADMILL-BASED EXERCISE TRAINING IMPROVES GAIT FUNCTION}

The initial studies with human SCI and subacute stroke subjects used TM training in conjunction with partial body-weight suspension (PBWS). In a randomized study of more severely impaired subjects with subacute stroke, Barbeau and Visintin found TM with PBWS to be more effective than TM without PBWS for improving selected mobility outcomes in those subjects with more severe motor deficits (i.e., $<0.2 \mathrm{~m} / \mathrm{s}$ walking velocity and Berg Balance scores <15) [24]. By week 6 of training, 79 percent of subjects were able to train at 0 percent PBWS. In a noncontrolled 3-week study, 25 PBWS TM training sessions improved mobility scores and gait temporal-distance parameters in nine nonambulatory stroke subjects [25]. PBWS was not required after day 6 of training in seven of these nine cases. Similar results were reported in a follow-up study using the same approach in an A-B-A design [26]. These studies indicate an important role for PBWS as a bridge to full-weightbearing TM exercise, particularly in subjects more severely affected. 
Other therapeutic approaches have been adapted from the original PBWS TM studies to include novel applications of robotically facilitated gait training with and without the TM and also with augmentation by functional electrical stimulation (FES). These approaches emphasize new gait therapies for nonambulatory patients with severe paresis after stroke or SCI. The Lokomat (Hocoma AG; Volketswil, Switzerland) is a robotic gait trainer that integrates PBWS and TM with actuated hip and knee orthoses to emulate normal walking patterns [27]. A recent randomized crossover study found that subjects with hemiparesis made greater improvements in gait function and lower-limb impairment measures following periods of Lokomat training compared with equal periods of conventional physical therapy [28]. Husemann et al. showed that 4 weeks of Lokomat and usual therapy improved the functional ambulation category for subjects in the acute phase of stroke as well as in those who received equal amounts of usual therapy only. However, the Lokomat group increased paretic single support times in overground walking, gained more muscle mass, and lost more fat compared with the controls, who gained fat mass [29]. Hesse et al. developed and tested an electromechanical gait trainer to move the legs in a manner physiologically similar to walking [25]. A study of survivors of stroke in the subacute phase of recovery showed that the electromechanical gait trainer was as effective as therapist-assisted PWBS TM training for improving gait function [26].

The gait trainer has also been used in conjunction with FES applied to knee extensors and ankle dorsiflexors in nonambulatory subjects with hemiparesis, for comparing the possible benefits of combined treatment versus either usual therapy or gait trainer alone in a 4-week intervention [30]. The gait trainer with FES group and gait trainer only group improved more than controls, but the two gait trainer methods did not differ. In another AB-A design study of a 9-week protocol, FES was used to augment PBWS TM therapy in a small sample of subjects with chronic stroke. Gait speed, cadence, and stride length increased significantly after the introduction of FES, and gait speed declined when FES was discontinued during a final phase of PBWS TM training only [31]. The potential for early intervention to enhance gait function by combining TM training with robotic assistance and/or FES is promising; however, further studies are needed to delineate optimal methods.
The question of how to optimize TM training for improving gait function after stroke is important but unsettled. We have investigated yet another approach to TM training during the chronic phase after stroke by emphasizing progressive cardiovascular demands over a 6-month program. Improved floor walking speeds, economy of gait, and cardiovascular fitness were reported for subjects with chronic hemiparesis after 6 months of TM aerobic exercise (T-AEX) training using handrail support without PBWS (Figures 1-2) [32]. A reference control group spent equivalent time performing stretching exercises. An important feature of the T-AEX protocol was the emphasis on aerobic conditioning, with increased TM walking duration and velocity to maintain 60 percent of heart rate reserve, a key indicator of exercise intensity [33-34]. While the primary focus was on cardiovascular conditioning, improvements were also found in fundamental gait parameters, indicating that T-AEX can differentially affect step lengths and walking cadence to achieve increased velocity [35]. As well, the double stance times

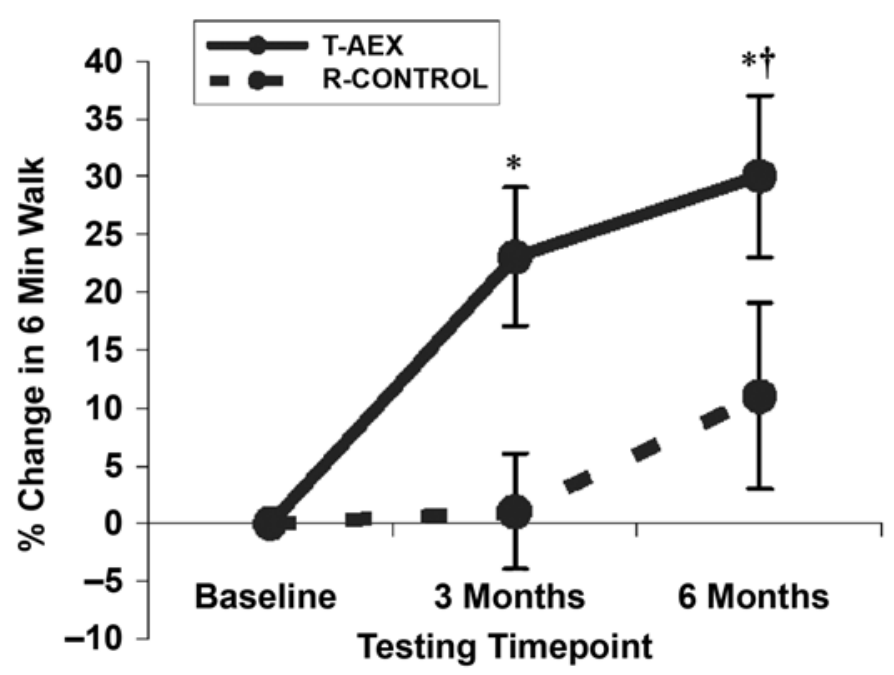

Figure 1.

Mean percent change in 6-minute walk distance in treadmill aerobic exercise (T-AEX) group (solid line) and reference control (R-CONTROL) stretching groups (dashed line). Significant groupby-time interaction occurred in 6 min walk distance by repeated measures analysis of variance $\left({ }^{\dagger} p<0.02\right)$ with progressive gains across 6-month intervention period $\left({ }^{*} p<0.05\right)$. Values are mean \pm standard error. Source: Reprinted by permission from Macko RF, Ivey FM, Forrester LW, Hanley D, Sorkin JD, Katzel LI, Silver $\mathrm{KH}$, Goldberg AP. Treadmill exercise rehabilitation improves ambulatory function and cardiovascular fitness in patients with chronic stroke: A randomized, controlled trial. Stroke. 2005;36(10): 2206-11. [PMID: 16151035] 


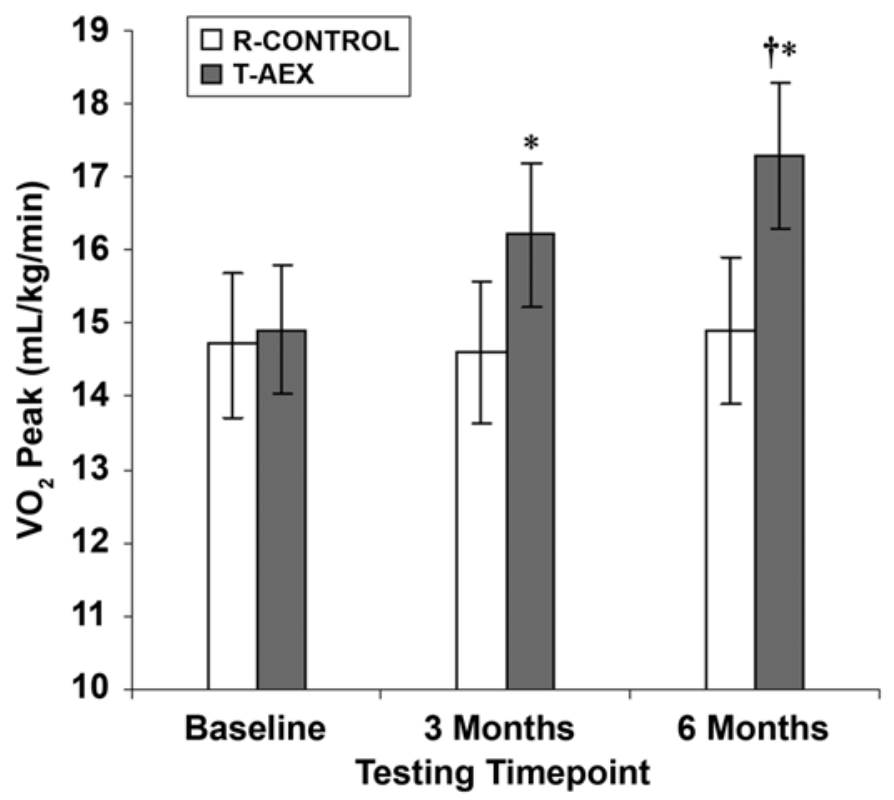

Figure 2.

Comparison between treadmill aerobic exercise (T-AEX) group and reference control (R-CONTROL) group for peak aerobic capacity across 6 months. Significant time by group interaction occurred in peak oxygen uptake $\left(\mathrm{VO}_{2}\right.$ peak) $(\mathrm{mL} / \mathrm{kg} / \mathrm{min})$ by repeated measures analysis of variance $\left({ }^{\dagger} p<0.005\right)$. $\mathrm{VO}_{2}$ peak was significantly different from baseline at both 3 - and 6-month time points within T-AEX $\left(^{*} p<\right.$ 0.05). Values are mean \pm standard error. Source: Reprinted from Macko RF, Ivey FM, Forrester LW, Hanley D, Sorkin JD, Katzel LI, Silver KH, Goldberg AP. Treadmill exercise rehabilitation improves ambulatory function and cardiovascular fitness in patients with chronic stroke: A randomized, controlled trial. Stroke. 2005;36(10):2206-11. [PMID: 16151035]

decreased, suggesting improved postural stability during weight shifts between the legs. Consideration of these changes in gait parameters and cardiovascular fitness together highlights the potential for T-AEX to translate improved gait function into capacities needed for sustained mobility in daily living and may help define clinically significant outcomes.

Other investigators have begun to focus on training intensity, which may involve manipulation of the velocity, duration, grade of incline, and concentration (massing) of practice. Sullivan et al. reported that after a 4-week PBWS training program, survivors of stroke who trained at faster TM velocities had greater increases in the criterion test of self-selected floor walking velocity [36]. In a randomized controlled trial, Pohl et al. systematically applied higher velocities to elicit greater improvements in overground walking parameters (velocity, cadence, stride length, func- tional ambulation category) after 4 weeks of TM training, compared with two reference groups receiving 20 percent speed increases or no speed increases [37]. Others are now looking at the use of TM grade to intensify the training stimulus, with one report showing increases in heart rate and improved gait pattern symmetry and longer stride lengths when grade is increased to 8 percent for subjects with hemiparesis [38]. In yet another variation of TM training, a split-belt TM altered hemiparetic gait patterning through differential belt speeds [39]. Adaptations as aftereffects in gait kinematics were observed in subjects with stroke, and these persisted briefly when subjects were transitioned immediately to overground walking. Although not yet proven durable, this observation suggests that the mechanical TM stimulus is affecting CNS motor planning of gait.

Few investigations have directly linked TM training to overground walking. Plummer et al. have been proponents of coupling PBWS TM training with transfer to the specific task of overground walking, immediately reinforced by cueing appropriate arm and stepping actions [40]. Their approach is grounded in neurally based functional requirements for walking, and their pilot data suggest that such an approach is safe and feasible for improving gait function among individuals who are moderately to severely impaired. We also have begun to look at the question of carryover from TM training to independent walking and report preliminary data on gait pattern changes after 6 months of T-AEX [35]. A key finding was that velocity improvements in unassisted 8-meter walks were due to a combination of increased stride length and frequency. Importantly, the training did not alter interlimb symmetry in either step times or step lengths; hence, both limbs appeared to amplify the preexisting hemiparetic pattern to improve overall gait function.

In the context of defining optimal training approaches, little is known about the interactions between deficit severity and any of these various training parameters. Individuals with stroke tend to have multiple comorbid conditions that can affect participation in TM training. This issue is now receiving closer attention. For example, in their pilot feasibility study, Plummer et al. stratified subjects with stroke according to self-selected walking velocity $(<0.4$ $\mathrm{m} / \mathrm{s}$ vs. $>0.4$ and $<0.8 \mathrm{~m} / \mathrm{s}$ ) [40]. The subjects who were moderately impaired made clinically meaningful gains after 24 sessions and the subjects who were severely impaired were improving, but not to a clinically meaningful level after the full 36-session program. This finding 
starts to provide a basis for constructing individualized therapy regimens based on ambulatory function.

Taken together, these studies indicate that concentrated practice through TM exercise training can improve gait function in survivors of subacute and chronic stroke. Mechanistically, they suggest that repetition of an effective gait pattern/rhythm may be critical to restoring gait function. However, also likely is that the long-term TM exercise affects a number of other processes besides learning a more functional gait pattern, including biological responses in peripheral muscle, balance control, and selfefficacy related to fall risk. Thus, a complete understanding of what transpires during any of these TM training regimens is very difficult to realize as we consider the potential mechanisms for improving hemiparetic gait. In the following sections, we focus on the emerging evidence that, like the upper limb, central neural plasticity is a likely mechanism underlying lower-limb functional recovery after stroke and that TM training can be a viable motorlearning stimulus for triggering that response.

\section{CNS ROLE IN LOWER-LIMB MOTOR CONTROL AND GAIT}

The neurophysiology of lower-limb motor control and its impact on locomotor recovery has become another focus for poststroke rehabilitation. Corticospinal connectivity to lower-limb musculature that determines ambulatory performance capacity is crucial to locomotor efficiency and recovery of basic activities of daily living. Studies of gait recovery after incomplete SCI and during normal motor development strongly suggest that improvement of human walking depends on enhanced cortical input [41]. In this section we summarize recent findings that employ a number of methods used in neurophysiological studies of upperlimb motor control to explore the central neural mechanisms of lower-limb motor control.

One noninvasive method to investigate lower-limb neurophysiology is transcranial magnetic stimulation (TMS), in which motor-evoked potentials (MEPs) are evaluated in the lower-limb musculature for characterizing aspects of the corticospinal connectivity that may underlie control of gait. Prolonged MEP latencies indicate descending pathway injury [42-43]. In the subacute phase of stroke, the ability to elicit lower-limb MEPs predicts improved long-term hemiparetic leg recovery [4445]. Like several studies that show significantly reduced MEP responses in the paretic arm or hand compared with responses in the nonparetic limb [46-49], TMS reveals decreased excitability to the paretic leg relative to the nonparetic leg [50]. These effects are noted mainly as increased motor thresholds, longer latencies, and reduced MEP amplitudes to paretic versus nonparetic quadriceps muscles. Furthermore, this effect was observed in individuals with a variety of lesion locations, illustrating the fundamental impairment of corticospinal connectivity associated with residual lower-limb weakness and hemiparetic gait.

A number of investigations with nondisabled individuals have used TMS to demonstrate the role of corticospinal connectivity in the control of walking. Using a specialized mounting apparatus to fix coil position, Schubert et al. applied TMS stimulations to the cortex during TM walking, showing that corticospinal excitability to ankle musculature was differentially affected by the phase of the gait cycle [51]. Additionally, excitability effects were substantially greater on dorsiflexors as compared with plantar flexors. Capaday et al. used a similar approach to administering TMS during TM walking and reinforced these findings, highlighting the importance of corticospinal connections to the tibialis anterior (TA) during swing phase, compared with relatively reduced MEP responses in the soleus during stance [52]. Several other studies from Bo Nielsen's group have elaborated on corticospinal contributions to gait [53]. Again, during active walking, TMS effects on $\mathrm{H}$ reflexes during the stance phase of the gait cycle were monitored to show that walking increases corticospinal excitability to ankle muscles, as evidenced by increases in $\mathrm{H}$ reflexes during walking but not under a controlled standing condition [54]. Furthermore, submotor threshold TMS delivered during walking caused suppression of the rectified EMG bursts from the TA and soleus muscles were suppressed, indicating that intracortical inhibitory responses were directly affecting the motor controlled of gait [55]. This protocol was modified to also show that long-latency stretch reflexes of the TA in nondisabled humans are at least partially modulated by transcortical circuits [56].

At least two studies have investigated brain activity during actual walking in nondisabled subjects. Fukuyama et al. used single photon emission computed tomography to show that several brain areas were active during overground walking in healthy subjects, including supplementary motor area (SMA), medial primary somatosensory cortex (S1), striatum, cerebellum, and visual cortex [57]. Activity across these distributed sites suggested that the brain is required to organize a complex flow of ongoing 
sensory and motor information during normal independent walking. Miyai et al. used near-infrared spectroscopy (NIRS) to show that walking and foot flexion cause bilateral primary motor cortex (M1) and SMA activation, compared with contralateral M1 foci during isolated arm movements [58]. Miyai et al. also extended this method to a small cohort of nonambulatory subjects with hemiplegia to characterize cortical responses during PBWS TM walking [59]. They employed two different modes of therapist assistance: one assisted the swing of the paretic leg directly and the other used pelvic maneuvers to facilitate paretic swing indirectly. In both modes, the NIRS maps indicated activation in the medial primary sensory motor cortices (SMCs), with more activity seen in the nonlesioned hemisphere. Enhanced activation of the premotor and presupplementary motor areas of the lesioned hemisphere were also observed during gait. The pelvic assistance method produced generally greater cortical activations compared with directly moving the paretic swing leg. While having the limitation of a small sample size, this study demonstrates the feasibility of using therapist-augmented PBWS TM exercise to engage cortical networks. The study suggests that different therapeutic strategies may have distinct effects on the CNS.

Another area of focus is determining the effects of differing sensory modalities on CNS activity. This relates to the role of feedback as a requirement for motor learning, and whether certain types and quantities of afferent information enhance or impede the learning process and neuroplasticity. In a manner similar to that for the upper limb [60], the cortical processing for lower-limb motor planning in nondisabled subjects adapts to increased sensory inputs by increasing recruitment of parietal, motor, and premotor areas [61]. Greater sensory demands from combined visual and proprioceptive modalities evoked increased movement-related cortical potentials (MRCPs) during performance of a knee extension task, compared with single modalities and unconstrained knee movements, which evoked the least activity (Figure 3). This increase in MRCP is encouraging because nonprimary motor areas are known to be involved in stroke motor recovery [17]. The increase suggests that rehabilitation strategies that use an enhanced sensory environment may induce greater activation along the neuraxis to mediate improved lower-limb function.

More broadly, these methods for instantiating the role of central neural processing in regulating motor activity related to normal lower-limb function also provide a basis for assessing how the CNS may adapt to exercise-mediated training in individuals with stroke. In the next section we examine findings of adaptations in CNS activity due to short-term exercise exposures.

\section{RAPID-TRANSIENT PLASTICITY IN LOWER LIMB}

Beyond investigating the nature of CNS activity in control of lower-limb muscles and gait function, noninvasive techniques have also revealed aspects of rapid CNS plasticity after brief exposures to motor practice. To a limited degree, these efforts parallel upper-limb studies that demonstrate the potential for rapid changes in CNS excitability and task-specific cortical activation in nondisabled and stroke populations. A seminal study by Classen et al. reported rapid plasticity in control of the thumb muscles in nondisabled subjects, as TMS to the same location caused the CNS to encode the opposite kinematic response after as little as 20 minutes of repetitive thumb exercises in the opposite direction [62].

Corticospinal responses to different modes of shortterm ankle exercise have been investigated in nondisabled subjects [63]. Recruitment curves from single-pulse TMS indicated that corticospinal excitability of the TA muscle increased after skill-based ankle training consisting of 32 minutes of volitional dorsi- and plantar flexion movements to track a target on a computer screen. Reference conditions with equal amounts of passive ankle movements or nonskilled volitional ankle movements did not show increased excitability. Another outcome was a decrease in intracortical inhibitory responses measured by paired-pulse TMS. Intracortical facilitation was not affected by the exercise. These results, along with no change on motor threshold levels and a negative finding in recruitment curves measured using transcranial electrical stimulation, were interpreted to suggest that the excitability changes due to skill-based exercise occurred at the cortical level.

Paired associative stimulation (PAS) has been used to investigate bidirectional corticospinal excitability of the hand muscles [64] by applying peripheral nerve stimulation to activate sensorimotor cortex within specified time windows around a pulse of TMS. When the afferent signals arrive at the cortex slightly ahead of the TMS impulse, excitability of the efferent pathways is enhanced. A recent study with nondisabled subjects examined the effects of PAS on TA responses during and following a 20-minute bout of TM walking at a moderate velocity $(1.1 \mathrm{~m} / \mathrm{s})$ [65]. 

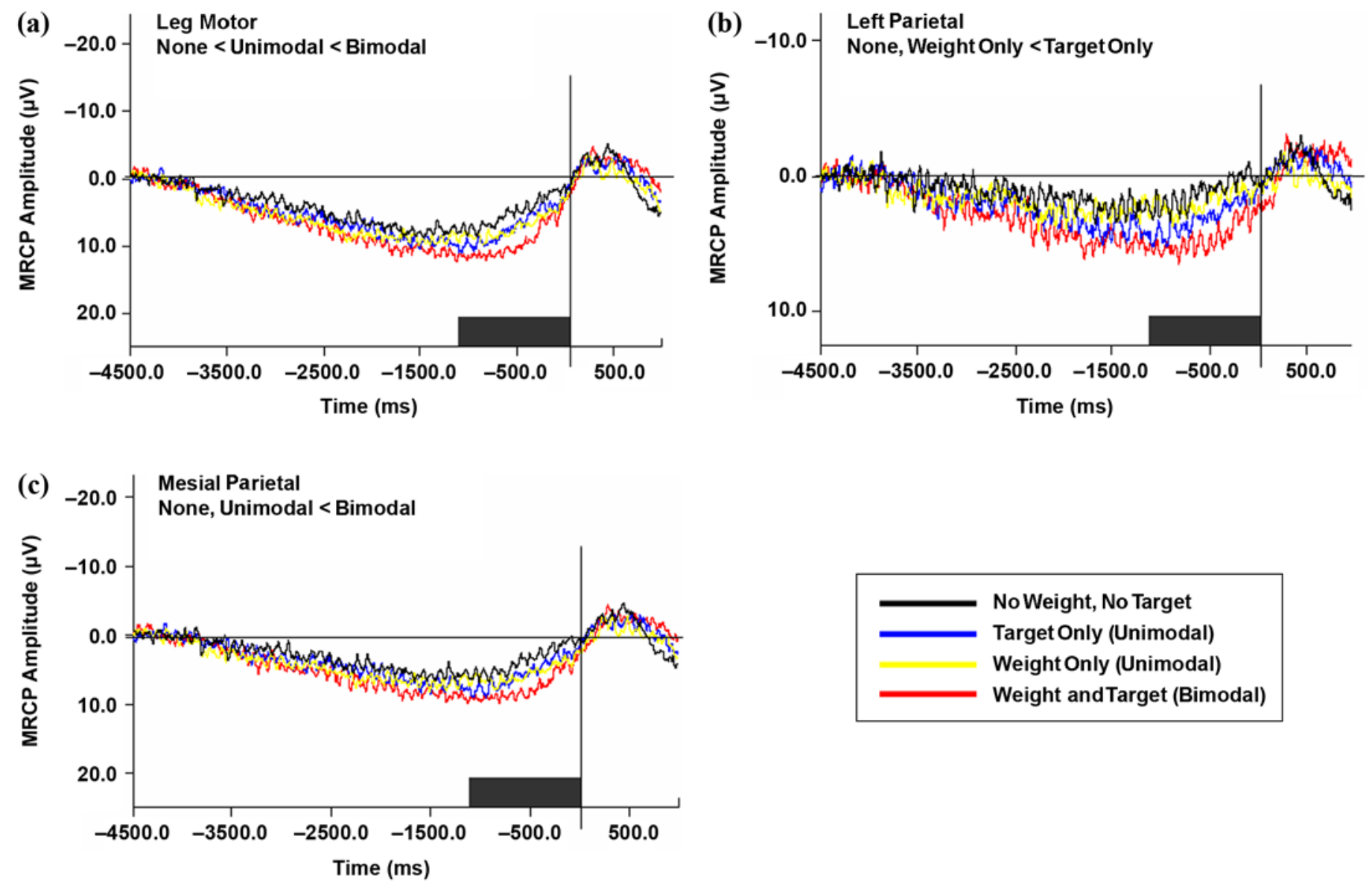

Figure 3.

Electroencephalographic recordings of movement-related cortical potentials (MRCPs) during four knee extension tasks: movement to visual target, with added $3.2 \mathrm{~kg}$ weight, with both target and weight, and with no target or weight. (a) Leg motor area shows increased activation related to increased numbers of sensory modality. However, (b) left parietal area appears to have increased activation based on presence of target and likely relates to increased visual processing demands. In addition, (c) mesial parietal area showed most activity in most complex condition (both target and weight). This effect is likely due to cingulate activity and needs to integrate more demanding task components. Black box indicates time bin of data analysis, and vertical line represents electromyography onset. Source: Adapted by permission from Wheaton LA, Mizelle JC, Forrester LW, Bai O, Shibasaki H, Macko RF. How does the brain respond to unimodal and bimodal sensory demand in movement of lower extremity? Exp Brain Res. 2007;180(2):345-54. [PMID: 17256159]

When peroneal nerve stimulation was timed to reach SMC approximately $5 \mathrm{~ms}$ before TMS and during the swing phase of ongoing TM walking, the posttest MEPs at the TA were significantly enhanced. When the TMS was administered before arrival of the afferent volley during walking, the posttest MEP amplitudes decreased compared with baseline. These results provide further evidence that sensory activation plays a key role in mediating CNS plasticity, which may be useful in rehabilitation of lower-limb function. One other small pilot study has shown potential for using the PAS approach in a therapeutic context for individuals with chronic stroke [66]. Although gait train- ing exercise was not part of the 4-week intervention, PAS was applied 30 minutes a day for a total of 20 sessions. While the small subject sample showed mixed results on neurophysiological measures after the treatments, most subjects showed increased MEP amplitudes. Also, some participants improved in walking cadence and stride length. This improvement could indicate that PAS may augment experience-based plasticity mechanisms that mediate functional gains after task-oriented training. However, further investigations are needed to assess these potentials, as Uy et al. emphasize that only some of the functional and neurophysiological measures produced 
significant changes, likely because of the small sample size and differences in lesion characteristics [66].

The effects of TM exercise on the CNS in subjects with hemiparesis have also been studied to better delineate its potential impact on neural mechanisms underlying hemiparetic gait. One approach has been examining the short-term effects of submaximal TM walking on the corticospinal responses of leg muscles. In subjects with chronic stroke, changes in quadriceps excitability have been elicited with short-term exposure to self-selected TM walking [50]. Two groups of subjects with chronic hemiparesis, one that trained for 6 months in a T-AEX program and the other that was untrained, were tested with TMS before and immediately after 20 minutes of self-selected, comfortable pace TM walking. The trained group exhibited increased MEP amplitudes in paretic quadriceps, whereas the untrained group showed no change (Figure 4). In a separate study of untrained subjects with hemiparesis, this protocol was extended to include a second session of dosetime-matched stretching exercises for comparison of excitability responses with stretching versus TM walking [67]. The results of the cross-sectional study were replicated, because the submaximal TM walking had no significant effect on paretic MEP latencies or amplitudes, although the amplitudes tended to decrease in both legs. However, stretching elicited significantly larger nonparetic MEP amplitudes but with no change on the paretic side. This finding suggested that sensorimotor stimulation from stretching may have increased excitability in the former, with the possibility that longer or more intensive stretching could lead to a similar effect in the latter.

From these studies in nondisabled subjects and those with stroke, considerable evidence now exists that cortical and cortico-spinal control of the lower limbs and gait is modifiable in a short-term, transient manner. Whether such neurophysiological changes presage CNS plasticity as a viable target for long-term therapies remains to be seen. In the next section we review early results from studies that combine noninvasive measures of CNS activity associated with altered gait function.

\section{DURABLE LOWER-LIMB PLASTICITY AFTER STROKE}

Brain plasticity occurs with motor recovery after stroke. Longitudinal imaging and TMS mapping studies clearly show that de novo sites of brain activation
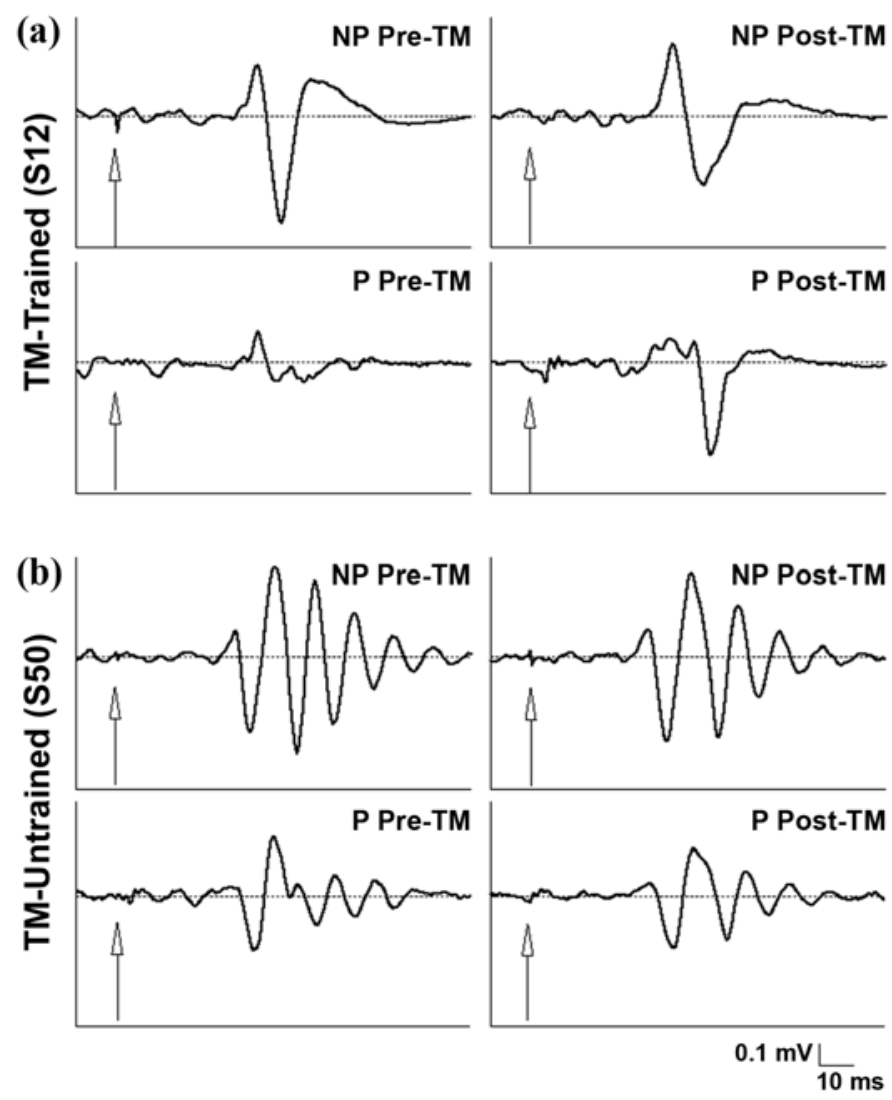

Figure 4.

Examples of 10 averaged transcranial magnetic stimulation-induced MEPs at vastus medialis before and after single session of treadmill (TM) walking exercise: (a) trained subject's nonparetic (NP) and paretic (P) responses and (b) responses of untrained subject. Arrows denote stimulus onset. $\mathrm{P}=$ paretic side, $\mathrm{S} 12$ = subject $12, \mathrm{~S} 50=$ subject 50. Source: Reprinted by permission from Forrester LW, Hanley DF, Macko RF. Effects of treadmill training on transcranial magnetic stimulation-induced excitability to quadriceps after stroke. Arch Phys Med Rehabil. 2006; 87(2):229-34. [PMID: 16442977]

become established in perilesional regions, as well as more remote areas of cortex and subcortical structures.

Upper-limb studies suggest that the lesioned hemisphere can affect cortico-muscular pathways, as repetitive TMS of the dominant, affected (but not the nondominant, unaffected) hemisphere impairs motor function to the affected hand [68]. One mechanism that may explain this control of the perilesional cortex is continued use of the affected limb, which may help maintain viable networks in the injured cortex [69-70]. Ipsilesional cortical activation has been shown to be a feature of locomotor recovery without specific training regimens [71]. Also, it is possible for cortical injury to prompt formation of axon projections 
to other cortical areas, which may promote reorganization via remodeled connections to cortical and subcortical structures [72-74].

The resultant patterns of brain reorganization after stroke appear to be strongly influenced by lesion location. Using functional magnetic resonance imaging (fMRI) techniques to study brain activations during knee movements, Luft et al. found differences in regional activations of the paretic limb versus the nonparetic limb in subjects with stroke and compared with nondisabled controls [75]. As seen with the upper limb [76], these analyses demonstrate heterogeneous CNS reorganization for lower-limb control that correlates to lesion location (Figures 5-6). Specifically, paretic knee motor control differed among survivors of stroke, such that subcortical strokes did not shift the locus of control away from M1, whereas cortical lesions induced shifts to more perilesional and contralateral control sites. Relationships to better walking function also varied by lesion location. Faster walking among subjects with brain stem lesions required lower ipsilesional M1 activity, whereas in subjects with subcortical strokes faster walking was linked to more activity in the contralesional versus ipsilesional SMC. For those with cortical lesions, faster walking was associated with increased activation in more widely distributed areas bilaterally, possibly signifying that greater compensations after cortical injury lead to better functional outcome. Future studies are needed with larger sample sizes to better define the possible links between severity of functional deficits and lesion location and whether they will indicate different rehabilitation strategies to optimize plasticity and locomotor function.

A key question then, given the apparent adaptability of the brain for lower-limb control after stroke, is whether and/or how this process can be exploited to the individual's advantage for regaining independent mobility. Added context for the recovery of gait function is provided by a study of lower-limb EMG timing patterns to assess possible changes in motor control of hemiparetic walking after 10 weeks of physical and occupational therapies in the subacute phase poststroke [77]. While significant improvements were reported in measures of gait function, including walking velocity and indices of walking independence, no changes in EMG patterns were observed in TM tests performed at the same velocity at all time points. This finding suggests that the neuromotor control of the lower limb during walking was not reorganized by the usual therapies, which concentrated on use of
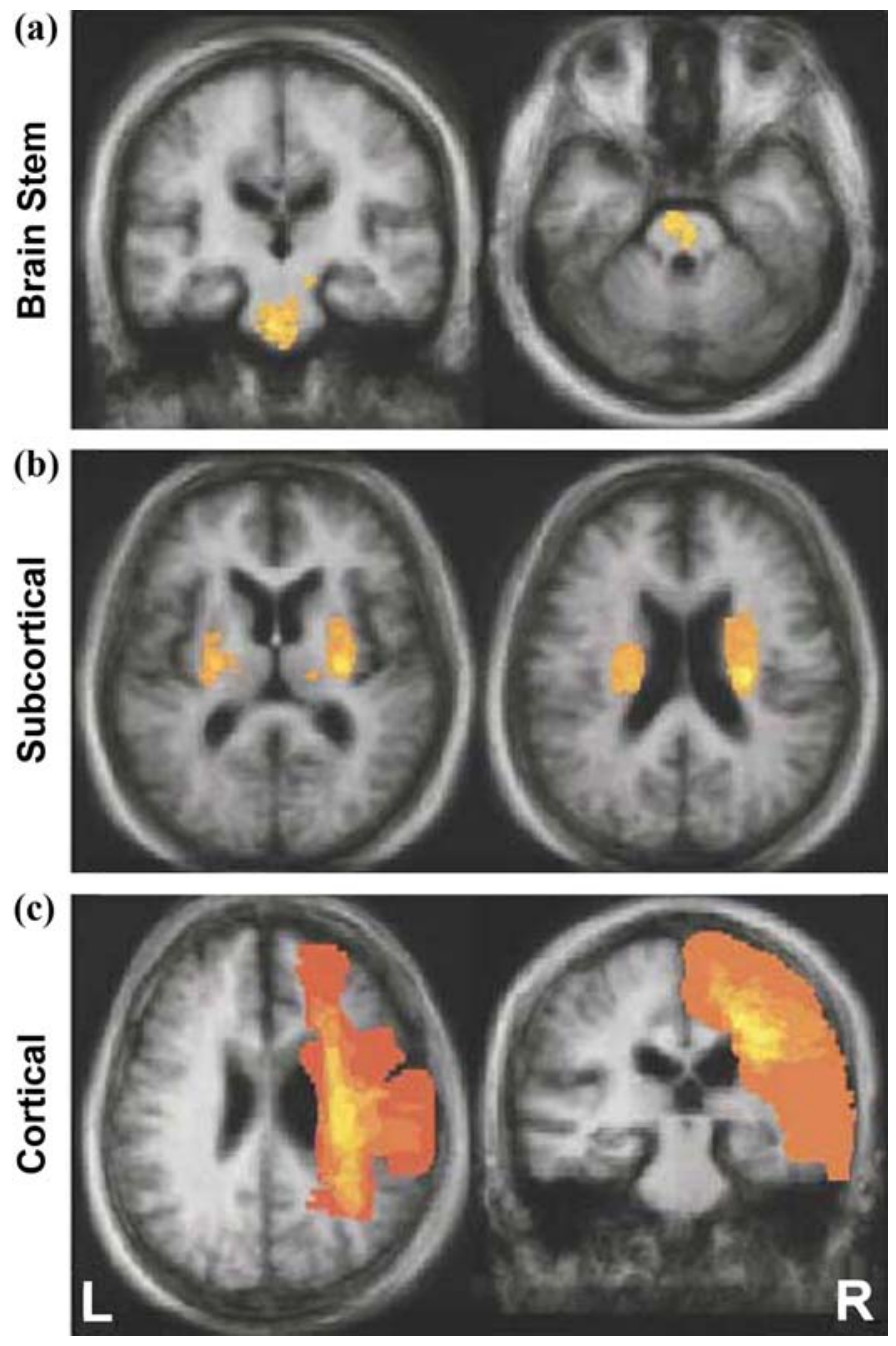

Figure 5.

For each group of subjects, average lesion distribution is superimposed onto averaged anatomical image. Shades of red to yellow indicate in how many of (a) 10 brain stem, (b) 12 subcortical, and (c) 9 cortical stroke subjects particular area was lesioned (red = injury less frequent, yellow = more frequent). $\mathrm{L}=$ left, $\mathrm{R}=$ right. Source: Reprinted by permission from Luft AR, Forrester L, Macko RF, McCombe-Waller S, Whitall J, Villagra F, Hanley DF. Brain activation of lower extremity movement in chronically impaired stroke survivors. Neuroimage. 2005;26(1):184-94. [PMID: 15862218]

the paretic limbs in the performance of gross motor skills and neurodevelopmental approaches. While Den Otter et al. concluded that locomotor functional gains could be elicited without concomitant changes in lower-limb muscle activity patterns, the results also suggest that taskspecificity of practice may be a precondition to altering the underlying motor control. The results also raise questions about whether the concentration of practice in the 

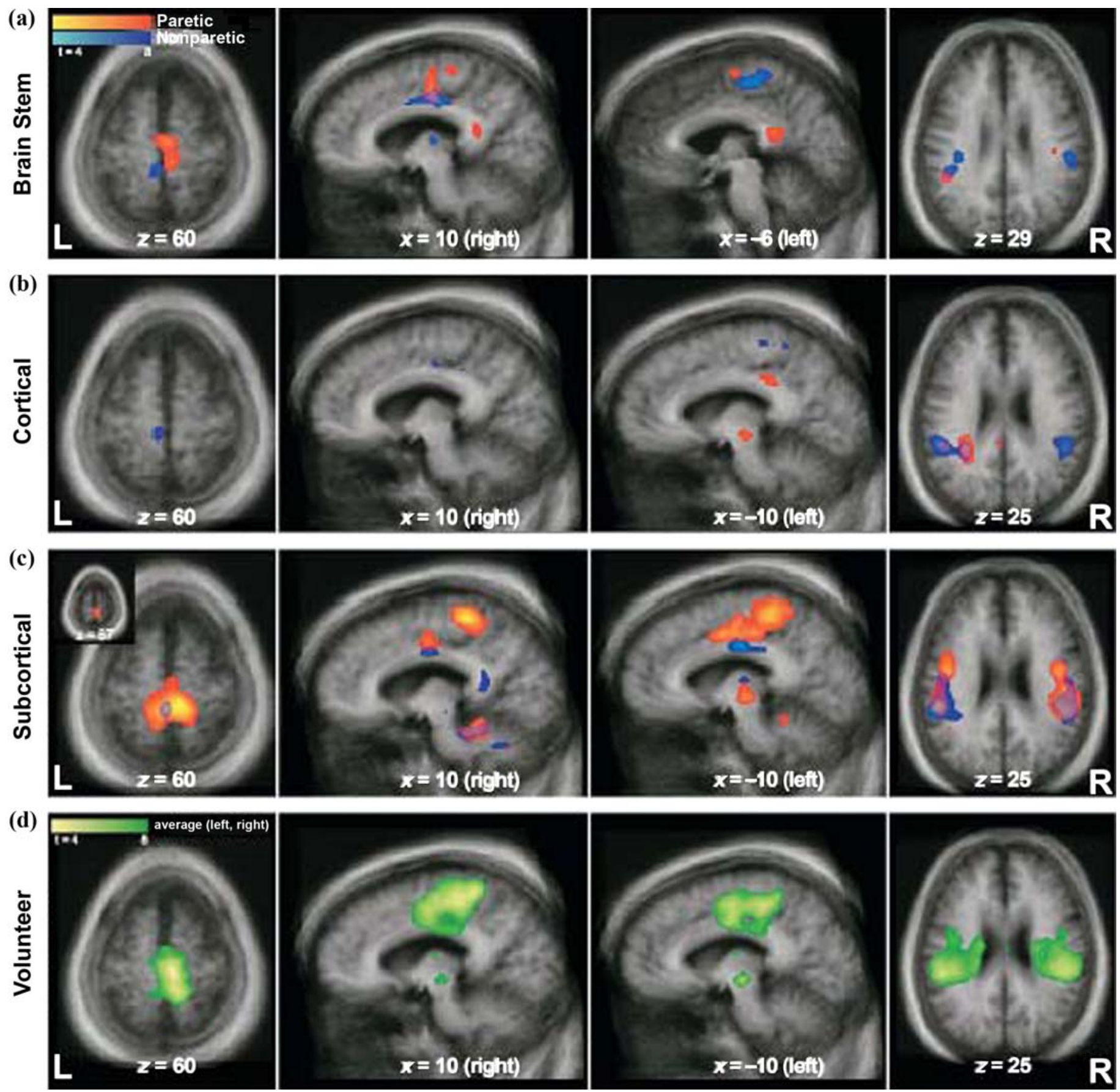

Figure 6.

For (a) brain stem, (b) cortical, (c) subcortical, and (d) nondisabled control subjects, activation patterns of paretic (red-yellow), nonparetic (blue), and nondisabled control knee movement (green) are superimposed onto averaged anatomical templates. Image data of subjects with left-sided stroke are flipped about midsagittal plane so that lesioned hemisphere is always on right. (d) For nondisabled control subjects, activation patterns of left- and right-sided knee movement were averaged (after appropriate flipping so that moving limb is on left). Whereas during paretic limb movement, (c) subjects with subcortical stroke and, to lesser degree, (a) brain stem subjects recruited sensorimotor cortex and supplementary motor area bilaterally, (b) almost no cortical activation is observed in subjects with cortical stroke. For nonparetic limb movement, consistent contralateral primary motor cortex activation is seen in all groups, but also markedly different from control. $\mathrm{L}=$ left side, $\mathrm{R}=$ right side. Source: Reprinted by permission from Luft AR, Forrester L, Macko RF, McCombe-Waller S, Whitall J, Villagra F, Hanley DF. Brain activation of lower extremity movement in chronically impaired stroke survivors. Neuroimage. 2005;26(1):184-94. [PMID: 15862218] 
usual therapy sessions was sufficient to promote adaptations due to motor learning [77].

To date, few neuroimaging studies exist of brain activation responses secondary to sustained intensive training of lower-limb motor function. However, evidence suggests that sufficient motor practice can alter CNS control of the lower limb and gait. For example, a case study by Carey et al. used fMRI to show the feasibility of promoting brain plasticity and durable functional benefits from visuomotor training of the paretic ankle [78]. The subject was trained to use a visual tracking system to monitor volitional dorsiplantar flexions of the paretic ankle during fMRI scans. After 16 sessions over a 4-week period, brain activation increased significantly, along with observed improvements in walking and ankle movements. Although these motor improvements were within the criterion difference of 2 standard deviations away from the baseline means, they were retained 4 months following completion of training.

Another fMRI study with four chronic survivors of stroke examined responses in cortical activity associated with ankle dorsiflexion control and lower-limb function during and after a 10-week program of PBWS TM training [79]. Serial fMRI tests were conducted at 2-week intervals, as were lower-limb Fugl-Meyer scores and walking velocity through 8 weeks of the protocol. The training produced increased activation areas in S1 and M1 regions, while functional performances improved. As function plateaued, the fMRI signals declined, a possible early indicator of learning consolidation.

Added perspective on the effects of PBWS on the CNS is gained from consideration of locomotor therapy in patients with incomplete SCI. Winchester et al. found that subjects with motor-incomplete SCI improved overground walking function that was associated with increased SMC and cerebellar activity after 12 weeks of PBWS TM training on the Lokomat robotic orthosis system [80]. Using intermuscular EMG coherence measures and TMS, Norton and Gorassini showed that training responses of incomplete SCI patients after 4 months of PBWS TM training depended on the extent of spared efferent pathways to the lower limbs [81]. The responders showed improved corticospinal connectivity in terms of increased EMG coherence at frequencies mediated by supraspinal inputs, as well as increased TMS MEP responses in the same muscles. For stroke, these improvements suggest that the degree of injury to descending pathways may have a significant effect on the capacity for the CNS plasticity to alter locomotor function, even with long-term training.
Miyai et al. conducted an intervention to study the effects of inpatient rehabilitation on eight patients who had not regained ambulatory function after 2 to 3 months of usual therapies following stroke [82]. Cortical activity was measured with NIRS during a standardized TM walking test conducted before and after a 2-month intervention that was based on a multidisciplinary neurodevelopmental approach. The regional activity changes detected from preand post-NIRS scans of subjects while walking showed improved symmetry in the medial primary SMCs from increased activation in the lesioned hemisphere and a reduction in the nonlesioned hemisphere. This finding parallels patterns of shifting cortical activation from nonlesioned to lesioned hemispheres in some studies of upperlimb recovery [83-84]. The change in the SMC laterality index also was significantly correlated to improved swingphase symmetry during the posttherapy walking trials. Other activation gains were seen in the lesioned side premotor area, whereas changes in laterality of the premotor and SMAs were not significant. Perhaps the most intriguing aspect of this study was that the adaptations in CNS locomotor control resulted from interventions that were not explicitly related to gait. While the plasticity of central neural control is evident, we cannot discern the relative contributions of ongoing recovery and the therapeutic intervention.

More recent evidence suggests that an intensive practice and training regimen of T-AEX training does modify brain areas controlling the paretic leg. A preliminary report on the effects of 6 months of T-AEX training on brain maps indicates strongly that subcortical structures, including bilateral red nucleus, represent new sites of paretic knee activation using the same fMRI knee protocols [85]. Correlations between changes in both voxelbased and region of interest analyses to changes in gait peak effort walking velocity appear to support functional relevance to the new areas of activity. If pending randomized controlled trial results confirm this effect, it will suggest that extensive massed practice on the TM may stimulate motor learning and foster new or reactivate unused bilateral pathways to mediate changes in gait, with the brain stem regions assuming a prominent role in remodeling the neuromotor coupling process.

\section{CONCLUSIONS}

The recent advances in motor-learning-based therapies have opened new possibilities for recovery of motor 
functions after stroke. The biology of central neural plasticity has emerged as a prime mechanism that may be exploited to optimize therapy for hemiparesis in the lower limb. In the area of gait rehabilitation, various methods of TM training have effectively improved walking function among individuals with hemiparesis following stroke. That such improvements can be achieved long after the expected time window for natural recovery supports the idea that the TM stimulus can promote motor learning and neuroplasticity of the lower limb. Considerable evidence now exists that supraspinal activity in the CNS, including interconnections among cortical, subcortical, and cerebellar pathways, plays a significant role in the control of lowerlimb movements and gait. These direct neurophysiological findings complement imaging studies showing that natural recovery with standard therapy does foster CNS plasticity of lower-limb motor control, similar to that reported for the upper limb [75].

Although stroke often affects motor function via injured supraspinal circuitry, lesion location and size have varied effects on lower-limb function including gait, especially if the pathways from the SMC leading to and through the reticulospinal areas are selectively affected. Deficient supraspinal input to the descending tracts may cause maladaptive plasticity within the spinal level circuitry. Stroke-induced loss of cortical inhibition over spinal reflex circuits can lead to a range of "upper motor neuron” signs, including clonus, positive extensor plantar reflexes, and spasticity. Sheean suggests that these signs are due to gradual and detrimental plasticity within the spinal cord, because these changes do not appear immediately after stroke [86]. Although individual responses to TM or other motor-learning-based rehabilitation programs (e.g., robotics) may differ according to level and size of infarct, no studies to date have reported distinctions in training efficacy related to these anatomical variables and whether or to what degree spinal centers would adapt.

We hypothesize that TM or other similar locomotor training that evokes functional improvement in gait through massed practice and goal-based progressions is likely to encourage positive rather than negative adaptation at the subcortical and/or spinal levels. Our recent results show that increased peak walking velocities are associated with new subcortical and cerebellar areas becoming active in paretic knee control [85]. Although individual responses to TM or other motor-learningbased rehabilitation programs (e.g., robotics) may differ according to level and size of infarct, this finding sug- gests that locomotor training may not need to be overly specific to foster benefits across different stroke subtypes. That said, we are far from having the means to determine whether a given survivor of stroke is a good or bad candidate for TM therapy, as substantial differences exist in gait deficit severity among survivors of stroke [40]. More rigorous study of factors such as lesion location, size, and the associated deficit profiles are needed to develop a sound clinical basis for prescribing and implementing individualized rehabilitation programs.

In line with established models of CNS plasticity, solid indications exist that the neuroplasticity associated with improved locomotor control may be facilitated if (1) the paretic leg is actively engaged in movement practice, (2) the practice includes high volumes of repetition, and (3) the practiced movements are task-relevant with an element of problem solving (e.g., focusing on specific elements of paretic leg stepping). TM training that progresses the performance demands or goals would seem to meet these criteria, whether through gradual reductions in PBWS or increasing practice workloads through longer duration and faster velocity or immediately transferring TM practice patterns to overground walking. Evidence of short-term adaptations in lower-limb neurophysiology support the possibility of modifying the neural control of hemiparetic gait through such training regimens. However, our understanding of how to take advantage of this process is extremely limited, with most attention given to varying the structure of locomotor practice.

Critical to these suggestions is determining the generalizability of TM training after stroke. We do not know how soon after stroke TM therapy should be started to optimize responses for lasting and clinically meaningful improvements in gait function. While we do know that individuals with disparate lesion locations and severity of locomotor deficits can benefit from TM exercise training, we still have very limited knowledge on how to tailor programs to specific cases. Current studies are looking at the relative effects of duration-based versus velocity-based approaches to TM training progressions on functional outcomes and CNS plasticity. On a broader note, and regardless of what technological advances eventually become efficacious, we now know that an opportunity exists to change the course of lower-limb recovery after hemiparetic stroke. Further studies are needed to determine optimal motor-learning strategies and dose intensities to improve mobility function poststroke. 


\section{ACKNOWLEDGMENTS}

This material was based on work supported in part by the Department of Veterans Affairs (VA) Rehabilitation Research and Development (RR\&D) Advanced Career Development Award B3390K to Dr. Larry Forrester and by a VA RR\&D Stroke Research Enhancement Award Program Fellowship to Dr. Richard Macko (which also funded work by Dr. Lewis Wheaton).

The authors have declared that no competing interests exist.

\section{REFERENCES}

1. Rosamond W, Flegal K, Friday G, Furie K, Go A, Greenlund K, Haase N, Ho M, Howard V, Kissela B, Kittner S, LloydJones D, McDermott M, Meigs J, Moy C, Nichol G, O’Donnell CJ, Roger V, Rumsfeld J, Sorlie P, Steinberger J, Thom T, Wasserthiel-Smoller S, Hong Y. Heart disease and stroke statistics - 2007 update: A report from the American Heart Association Statistics Committee and Stroke Statistics Subcommittee. Circulation. 2007;115:e69-e171.

2. Gresham GE, Fitzpatrick TE, Wolf PA, McNamara PM, Kannel WB, Dawber TR. Residual disability in survivors of stroke-The Framingham study. N Engl J Med. 1975; 293(19):954-56. [PMID: 1178004$]$

3. Gresham GE, Phillips TF, Wolf PA, McNamara PM, Kannel WB, Dawber TR. Epidemiologic profile of long-term stroke disability: The Framingham study. Arch Phys Med Rehabil. 1979;60(11):487-91. [PMID: 508073]

4. Fisher SV, Gullickson G Jr. Energy cost of ambulation in health and disability: A literature review. Arch Phys Med Rehabil. 1978;59(3):124-33. [PMID: 148252]

5. Fujitani J, Ishikawa T, Akai M, Kakurai S. Influence of daily activity on changes in physical fitness for people with post-stroke hemiplegia. Am J Phys Med Rehabil. 1999; 78(6):540-44. [PMID: 10574169]

6. Olney SJ, Richards C. Hemiparetic gait following stroke. Part I: Characteristics. Gait Posture. 1996;4(2):136-48.

7. Richards C, Olney SJ. Hemiparetic gait following stroke. Part II: Recovery and physical therapy. Gait Posture. 1996; 4(2):149-62.

8. Jorgensen HS, Nakayama H, Raaschou HO, Olsen TS. Recovery of walking function in stroke patients: The Copenhagen Stroke Study. Arch Phys Med Rehabil. 1995; 76(1):27-32. [PMID: 7811170]

9. Wolpaw JR, Carp JS. Plasticity from muscle to brain. Prog Neurobiol. 2006;78(3-5):233-63. [PMID: 16647181]

10. Schmidt RA. Motor control and learning: A behavioral emphasis. 2nd ed. Champaign (IL): Human Kinetics; 1988.
11. Wolf SL, Winstein CJ, Miller JP, Taub E, Uswatte G, Morris D, Giuliani C, Light KE, Nichols-Larsen D; EXCITE Investigators. Effect of constraint-induced movement therapy on upper extremity function 3 to 9 months after stroke: The EXCITE randomized clinical trial. JAMA. 2006; 296(17):2095-2104. [PMID: 17077374$]$

12. Liepert J, Miltner WH, Bauder H, Sommer M, Dettmers C, Taub E, Weiller C. Motor cortex plasticity during constraintinduced movement therapy in stroke patients. Neurosci Lett. 1998;250(1):5-8. [PMID: 9696052]

13. Liepert J, Bauder H, Wolfgang HR, Miltner WH, Taub E, Weiller C. Treatment-induced cortical reorganization after stroke in humans. Stroke. 2000;31(6):1210-16. [PMID: 10835434]

14. Levy CE, Nichols DS, Schmalbrock PM, Keller P, Chakeres DW. Functional MRI evidence of cortical reorganization in upper-limb stroke hemiplegia treated with constraintinduced movement therapy. Am J Phys Med Rehabil. 2001; 80(1):4-12. [PMID: 11138954]

15. Wittenberg GF, Chen R, Ishii K, Bushara KO, Eckloff S, Croarkin E, Taub E, Gerber LH, Hallett M, Cohen LG. Constraint-induced therapy in stroke: Magnetic-stimulation motor maps and cerebral activation. Neurorehabil Neural Repair. 2003;17(1):48-57. [PMID: 12645445]

16. Whitall J, McCombe Waller S, Silver KH, Macko RF. Repetitive bilateral arm training with rhythmic auditory cueing improves motor function in chronic hemiparetic stroke. Stroke. 2000;31(10):2390-95. [PMID: 11022069]

17. Luft AR, McCombe-Waller S, Whitall J, Forrester LW, Macko R, Sorkin JD, Schultz JB, Goldberg AP, Hanley DF. Repetitive bilateral arm training and motor cortex activation in chronic stroke: A randomized controlled trial. JAMA. 2004;292(15):1853-61. [PMID: 15494583]

18. Brown TG. The intrinsic factors in the act of progression in the mammal. Proc R Soc Lond (Biol). 1973;84(572):308-19.

19. Visintin M, Barbeau $H$. The effects of body weight support on the locomotor pattern on spastic paretic patients. Can J Neurol Sci. 1989;16(3):315-25. [PMID: 2766124]

20. Visintin M, Barbeau H, Korner-Bitensky N, Mayo NE. A new approach to retrain gait in stroke patients through body weight support and treadmill stimulation. Stroke. 1998; 29(6):1122-28. [PMID: 9626282]

21. Dobkin BH. An overview of treadmill locomotor training with partial body weight support: A neurophysiologically sound approach whose time has come for randomized clinical trials. Neurorehabil Neural Repair. 1999;13(3):157-65.

22. Harris-Love ML, Forrester LW, Macko RF, Silver KH, Smith GV. Hemiparetic gait parameters in overground versus treadmill walking. Neurorehabil Neural Repair. 2001; 15(2):105-12. [PMID: 11811252]

23. Harris-Love M, Macko RF, Whitall J, Forrester LW. Improved hemiparatic muscle activation in treadmill versus overground walking. Neurorehabil Neural Repair. 2004;18: 154-60. 
24. Barbeau H, Visintin M. Optimal outcomes obtained with body-weight support combined with treadmill training in stroke subjects. Arch Phys Med Rehabil. 2003;84(10): 1458-65. [PMID: 14586912]

25. Hesse S, Bertelt C, Schaffrin A, Malezic M, Mauritz KH. Restoration of gait in nonambulatory hemiparetic patients by treadmill training with partial body-weight support. Arch Phys Med Rehabil. 1994;75(10):1087-93. [PMID: 7944913]

26. Werner C, Von Frankenberg S, Trieg T, Konrad M, Hesse S. Treadmill training with partial body weight support and an electromechanical gait trainer for restoration of gait in subacute stroke patients: A randomised crossover study. Stroke. 2002;33(12):2895-2901. [PMID: 12468788]

27. Colombo G, Joerg M, Schreier R, Dietz V. Treadmill training of paraplegic patients using a robotic orthosis. J Rehabil Res Dev. 2000;37(6):693-700. [PMID: 11321005]

28. Mayr A, Kofler M, Quirbach E, Matzak H, Fröhlich K, Saltuari L. Prospective, blinded, randomized crossover study of gait rehabilitation in stroke patients using the Lokomat gait orthosis. Neurorehabil Neural Repair. 2007;21(4):307-14. [PMID: 17476001]

29. Husemann B, Müller F, Krewer C, Heller S, Koenig E. Effects of locomotion training with assistance of a robotdriven gait orthosis in hemiparetic patients after stroke: A randomized controlled pilot study. Stroke. 2007;38(2): 349-54. [PMID: 17204680]

30. Ng MFW, Tong RKY, Li LSW. A pilot study of randomized clinical controlled trial of gait training in subacute stroke patients with partial body-weight support electromechanical gait trainer and functional electrical stimulation: Six-month follow-up. Stroke. 2008;39:154-60.

31. Lindquist AR, Prado CL, Barros RM, Mattioli R, Da Costa $\mathrm{PH}$, Salvini TF. Gait training combining partial bodyweight support, a treadmill, and functional electrical stimulation: Effects on poststroke gait. Phys Ther. 2007;87(9): 1144-54. [PMID: 17609334$]$

32. Macko RF, Ivey FM, Forrester LW, Hanley D, Sorkin JD, Katzel LI, Silver KH, Goldberg AP. Treadmill exercise rehabilitation improves ambulatory function and cardiovascular fitness in patients with chronic stroke: A randomized, controlled trial. Stroke. 2005;36(10):2206-11. [PMID: 16151035]

33. Macko RF, DeSouza CA, Tretter LD, Silver KH, Smith GV, Anderson PA, Tomoyasu N, Gorman P, Dengel DR. Treadmill aerobic exercise training reduces the energy expenditure and cardiovascular demands of hemiparetic gait in chronic stroke patients. A preliminary report. Stroke. 1997;28(2):326-30. [PMID: 9040684$]$

34. Macko RF, Smith GV, Dobrovolny CL, Sorkin JD, Goldberg AP, Silver KH. Treadmill training improves fitness reserve in chronic stroke patients. Arch Phys Med Rehabil. 2001;82(7):879-84. [PMID: 11441372]

35. Patterson SL, Rodgers MM, Macko RF, Forrester LW. Effect of treadmill exercise training on spatial and temporal gait parameters in subjects with chronic stroke: A preliminary report. J Rehabil Res Dev. 2008;45(2):221-28.

36. Sullivan KJ, Knowlton BJ, Dobkin BH. Step training with body weight support: Effect of treadmill speed and practice paradigms on poststroke locomotor recovery. Arch Phys Med Rehabil. 2002;83(5):683-91. [PMID: 11994808]

37. Pohl M, Mehrholz PT, Ritschel C, Ruckriem S. Speeddependent treadmill training in ambulatory hemiparetic stroke subjects: A randomized controlled trial. Stroke. 2002; 33(2):553-58. [PMID: 11823669]

38. Werner C, Lindquist AR, Bardeleben A, Hesse S. The influence of treadmill inclination on the gait of ambulatory hemiparetic subjects. Neurorehabil Neural Repair. 2007; 21(1):76-80. [PMID: 17172557]

39. Reisman DS, Wityk R, Silver K, Bastian AJ. Locomotor adaptation on a split-belt treadmill can improve walking symmetry post-stroke. Brain. 2007;130(7):1861-72.

40. Plummer P, Behrman AL, Duncan PW, Spigel P, Saracino D, Martin J, Fox E, Thigpen M, Kautz SA. Effects of stroke severity and training duration on locomotor recovery after stroke: A pilot study. Neurorehabil Neural Repair. 2007; 21(2):137-51. [PMID: 17312089]

41. Yang JF, Gorassini M. Spinal and brain control of human walking: Implications for retraining of walking. Neuroscientist. 2006;12(5):379-89. [PMID: 16957000]

42. MacDonnell RA, Donnan GA, Bladin PF. A comparison of somatosensory evoked and motor evoked potentials in stroke. Ann Neurol. 1989;25(1):68-73. [PMID: 2913930]

43. Misra UK, Kalita J. Motor evoked potential changes in ischaemic stroke depend on stroke location. J Neurol Sci. 1995;134(1-2):67-72. [PMID: 8747846]

44. Cruz Martinez A, Tejada J. Central motor conduction in hereditary motor and sensory neuropathy and hereditary spastic paraplegia. Electromyogr Clin Neurophysiol. 1999; 39(6):331-35. [PMID: 10499202]

45. Palliyath S. Role of central conduction time and motor evoked response amplitude in predicting stroke outcome. Electromyogr Clin Neurophysiol. 2000;40(5):315-20. [PMID: 10938999]

46. Koski L, Mernar TJ, Dobkin BH. Immediate and long-term changes in corticomotor output in response to rehabilitation: Correlation with functional improvements in chronic stroke. Neurorehabil Neural Repair. 2004;18(4):230-49. [PMID: 15537994]

47. Seitz RJ, Hoflich P, Binkofski F, Tellmann L, Herzog H, Freund HJ. Role of the premotor cortex in recovery from middle cerebral artery infarction. Arch Neurol. 1998; 55(8):1081-88. [PMID: 9708958] 
48. Thickbroom GW, Byrnes ML, Archer SA, Mastaglia FL. Motor outcome after subcortical stroke: MEPs correlate with hand strength but not dexterity. Clin Neurophysiol. 2002;113(12):2025-29. [PMID: 12464343]

49. Turton A, Lemon RN. The contribution of fast corticospinal input to the voluntary activation of proximal muscles in normal subjects and in stroke patients. Exp Brain Res. 1999;129(4):559-72. [PMID: 10638430]

50. Forrester LW, Hanley DF, Macko RF. Effects of treadmill training on transcranial magnetic stimulation-induced excitability to quadriceps after stroke. Arch Phys Med Rehabil. 2006;87(2):229-34. [PMID: 16442977]

51. Schubert M, Curt A, Jensen L, Deitz V. Corticospinal input in human gait: Modulation of magnetically evoked motor responses. Exp Brain Res. 1997;115(2):234-46. [PMID: 9224852]

52. Capaday C, Lavoie BA, Barbeau H, Schneider C, Bonnard M. Studies on the corticospinal control of human walking. I. Responses to focal transcranial magnetic stimulation of the motor cortex. J Neurophysiol. 1999;81(1):129-39. [PMID: 9914274]

53. Bo Nielsen J. Motoneuronal drive during human walking. Brain Res Brain Res Rev. 2002;40(1-3):192-201. [PMID: 12589917]

54. Petersen N, Christensen LO, Nielsen J. The effect of transcranial magnetic stimulation on the soleus $\mathrm{H}$ reflex during human walking. J Physiol. 1998;513(Pt 2):599-610. [PMID: 9807007]

55. Petersen NT, Butler JE, Marchand-Pauvert V, Fisher R, Ledebt A, Pyndt HS, Hansen NL, Nielsen JB. Suppression of EMG activity by transcranial magnetic stimulation in human subjects during walking. J Physiol. 2001;537(Pt 2):651-56. [PMID: 11731595]

56. Van Doornik J, Masakado Y, Sinkjaer T, Nielsen JB. The suppression of the long-latency stretch reflex in the human tibialis anterior muscle by transcranial magnetic stimulation. Exp Brain Res. 2004;157(3):403-6. [PMID: 15221177]

57. Fukuyama H, Ouchi Y, Matsuzaki S, Nagahama Y, Yamauchi H, Ogawa M, Kimura J, Shibasaki H. Brain functional activity during gait in normal subjects: ASPECT study. Neurosci Lett. 1997;228(3):183-86. [PMID: 9218638]

58. Miyai I, Tanabe HC, Sase I, Eda H, Oda I, Konishi I, Tsunazawa Y, Suzuki T, Yanagida T, Kubota K. Cortical mapping of gait in humans: A near-infrared spectroscopic topography study. Neuroimage. 2001;14(5):1186-92. [PMID: 11697950]

59. Miyai I, Yagura H, Oda I, Konishi I, Eda H, Suzuki T, Kubota K. Premotor cortex is involved in restoration of gait in stroke. Ann Neurol. 2002;52(2):188-94.

[PMID: 12210789$]$

60. Cui RQ, Deecke L. High resolution DC-EEG of the Bereitschaftspotential preceding anatomically congruent ver- sus spatially congruent bimanual finger movements. Brain Topogr. 1999;12(2):117-27. [PMID: 10642011]

61. Wheaton LA, Mizelle JC, Forrester LW, Bai O, Shibasaki $\mathrm{H}$, Macko RF. How does the brain respond to unimodal and bimodal sensory demand in movement of lower extremity? Exp Brain Res. 2007;180(2):345-54. [PMID: 17256159]

62. Classen J, Liepert J, Wise SP, Hallet M, Cohen LG. Rapid plasticity of human cortical movement representation induced by practice. J Neurophysiol. 1998;79(2):1117-23. [PMID: 9463469]

63. Perez MA, Lungholt BK, Nyborg K, Nielsen JB. Motor skill training induces changes in the excitability of the leg cortical area in healthy humans. Exp Brain Res. 2004; 159(2):197-205. [PMID: 15549279]

64. Stefan KE, Kunesch E, Cohen LG, Benecke R, Classen J. Induction of plasticity in the human motor cortex by paired associative stimulation. Brain. 2000;123(Pt 3):572-84. [PMID: 10686179]

65. Stinear JW, Hornby TG. Stimulation-induced changes in lower limb corticomotor excitability during treadmill walking in humans. J Physiol. 2005;567(Pt 2):701-11. [PMID: 15975980]

66. Uy J, Ridding MC, Hillier S, Thompson PD, Miles TS. Does induction of plastic change in motor cortex improve leg function after stroke? Neurology. 2003;61(7):982-84. [PMID: 14557574]

67. Forrester LW, Villagra F, Macko RF, Hanley DF. Treadmill vs. stretching: Short-term CNS adaptations to single bouts of submaximal exercise in chronic stroke patients. Stroke. 2004;35(6):e312.

68. Fridman EA, Hanakawa T, Chung M, Hummel F, Leiguarda RC, Cohen LG. Reorganization of the human ipsilesional premotor cortex after stroke. Brain. 2004;127(Pt 4):747-58. [PMID: 14749291]

69. Friel KM, Heddings AA, Nudo RJ. Effects of postlesion experience on behavioral recovery and neurophysiologic reorganization after cortical injury in primates. Neurorehabil Neural Repair. 2000;14(3):187-98. [PMID: 11272475]

70. Heddings AA, Friel KM, Plautz EJ, Barbay S, Nudo RJ. Factors contributing to motor impairment and recovery after stroke. Neurorehabil Neural Repair. 2000;14(4):301-10. [PMID: 11402880]

71. Kim YH, You SH, Kwon YH, Hallett M, Kim JH, Jang SH. Longitudinal fMRI study for locomotor recovery in patients with stroke. Neurology. 2006;67(2):330-33. [PMID: 16864832]

72. Papadopoulos CM, Tsai SY, Alsbiei T, O’Brien TE, Schwab ME, Kartje GL. Functional recovery and neuroanatomical plasticity following middle cerebral artery occlusion and IN-1 antibody treatment in the adult rat. Ann Neurol. 2002;51(4):433-41. [PMID: 11921049] 
73. Frost SB, Barbay S, Friel KM, Plautz EJ, Nudo RJ. Reorganization of remote cortical regions after ischemic brain injury: A potential substrate for stroke recovery. J Neurophysiol. 2003;89(6):3205-14. [PMID: 12783955$]$

74. Dancause N, Barbay S, Frost SB, Plautz EJ, Chen D, Zoubina EV, Stowe AM, Nudo RJ. Extensive cortical rewiring after brain injury. J Neurosci. 2005;25(44):10167-79. [PMID: 16267224$]$

75. Luft AR, Forrester L, Macko RF, McCombe-Waller S, Whitall J, Villagra F, Hanley DF. Brain activation of lower extremity movement in chronically impaired stroke survivors. Neuroimage. 2005;26(1):184-94. [PMID: 15862218]

76. Luft AR, Waller S, Forrester LW, Smith GV, Whitall J, Macko RF, Schulz JB, Hanley DF. Lesion location alters brain activation in chronically impaired stroke survivors. Neuroimage. 2004;21(3):924-35. [PMID: 15006659]

77. Den Otter AR, Geurts AC, Mulder T, Duysens J. Gait recovery is not associated with changes in the temporal patterning of muscle activity during treadmill walking in patients with post-stroke hemiparesis. Clin Neurophysiol. 2006;117(1): 4-15. [PMID: 16337186]

78. Carey JR, Anderson KM, Kimberley TJ, Lewis SM, Auerbach EJ, Ugurbil K. fMRI analysis of ankle movement tracking training in subject with stroke. Exp Brain Res. 2004; 154(3):281-90. [PMID: 14578998]

79. Dobkin BH, Firestine A, West M, Saremi K, Woods R. Ankle dorsiflexion as an fMRI paradigm to assay motor control for walking during rehabilitation. Neuroimage. 2004; 23(1):370-81. [PMID: 15325385]

80. Winchester P, McColl R, Querry R, Foreman N, Mosby J, Tansey K, Williamson J. Changes in supraspinal activation patterns following robotic locomotor therapy in motorincomplete spinal cord injury. Neurorehabil Neural Repair. 2005;19(4):313-24. [PMID: 16263963]

81. Norton JA, Gorassini MA. Changes in cortically related intermuscular coherence accompanying improvements in locomotor skills in incomplete spinal cord injury. J Neurophysiol. 2006;95(4):2580-89. [PMID: 16407422]

82. Miyai I, Yagura H, Hatakenaka M, Oda I, Konishi I, Kubota K. Longitudinal optical imaging study for locomotor recovery after stroke. Stroke. 2003;34(12):2866-70. [PMID: 14615624$]$

83. Calautti CF, Leroy F, Guincestre JY, Marie RM, Baron JC. Sequential activation brain mapping after subcortical stroke: Changes in hemispheric balance and recovery. Neuroreport. 2001;12(18):3883-86. [PMID: 11742203]

84. Cramer SC, Nelles G, Benson RR, Kaplan JD, Parker RA, Kwong KK, Kennedy DN, Finklestein SP, Rosen BR. A functional MRI study of subjects recovered from hemiparetic stroke. Stroke. 1997;28(12):2518-27. [PMID: 9412643]

85. Luft AR, Macko R, Forrester L, Villagra F, Hanley D. Subcortical reorganization induced by aerobic locomotor training in chronic stroke survivors. Program No. 865.8. 2005 Abstract Viewer/Itinerary Planner. Washington, DC: Society for Neuroscience; 2005. Online.

86. Sheean G. The pathophysiology of spasticity. Eur J Neurol. 2002;9 Suppl 1:3-9;discussion 53-61. [PMID: 11918643]

Submitted for publication February 14, 2007. Accepted in revised form November 19, 2007. 